\title{
Spectral Analysis on Commutative Hypergroups
}

\author{
László Székelyhidi \\ Institute of Mathematics, University of Debrecen, \\ e-mail: lszekelyhidi@gmail.com \\ László Vajday \\ Institute of Mathematics, University of Debrecen, \\ e-mail: laszlo.vajday@gmail.com
}

\begin{abstract}
The problem of spectral analysis is formulated on commutative hypergroups and is solved for finite dimensional varieties.
\end{abstract}

\section{Introduction}

The study of spectral analysis and spectral synthesis problems is based on the concept of exponential monomials. Unfortunately at this moment we do not have a general definition of this concept on arbitrary (commutative) hypergroups hence on each special type of hypergroups we need to introduce the most appropriate form. In the papers [1] and [2] we have seen the corresponding definition on polynomial hypergroups in one variable and in several variables, respectively. Using these concepts we were able to prove spectral analysis and spectral synthesis on these types of hypergroups. Here we deal with spectral analysis only and we prove it for finite dimensional varieties on commutative hypergroups hence we need exclusively the concept of exponentials.

Let $K$ be a commutative hypergroup. The continuous function $m: K \rightarrow \mathbb{C}$ is an exponential if

$$
m(x * y)=m(x) m(y), \quad m(0)=1
$$

holds for any $x, y$ in $K$.

The research was supported by the Hungarian National Foundation for Scientific Research (OTKA), Grant No. NK-68040.

Keywords and phrases: spectral analysis, spectral synthesis, hypergroups

AMS (2000) Subject Classification: 43A45, 20N20 
If $y$ is in $K$ and $f: K \rightarrow \mathbb{C}$ is a continuous function, then the function $\tau_{y} f$ defined by

$$
\tau_{y} f(x)=f(x * y)
$$

for each $x$ in $K$ is called the translate of $f$ by $y$. Let $\mathcal{C}(K)$ denote the space of all continuous complex valued functions on $K$ equipped with the pointwise operations and the topology of uniform convergence on compact sets. A closed subspace $V$ of $\mathcal{C}(K)$ is called a variety, if it is translation invariant, that is, if $f$ belongs to $V$ then all translates of $f$ belongs to $V$, too.

The problem of spectral analysis for a given variety means that we are looking for exponentials in the variety. If there is an exponential in the variety then we say that spectral analysis holds for the variety. If spectral analysis holds for each nonzero variety, then we say that spectral analysis holds on the hypergroup $K$. For instance - as we have mentioned above - spectral analysis holds on polynomial hypergroups.

\section{Spectral analysis for finite dimensional vari- eties}

Theorem 1. Spectral analysis holds for nonzero finite dimensional varieties on every commutative hypergroup.

Proof. Suppose that $K$ is a commutative hypergroup and $V \neq\{0\}$ is a finite dimensional variety in $\mathcal{C}(K)$. We have to show that $V$ contains an exponential.

Let $f_{1}, f_{2}, \ldots, f_{n}$ be a basis of $V$, then there exist complex valued funcions $c_{i, j}$ for $i, j=1,2, \ldots, n$ such that

$$
f_{i}(x * y)=\sum_{j=1}^{n} c_{i, j}(y) f_{j}(x)
$$

holds for every $x, y$ in $K$ and $i=1,2, \ldots, n$. As the functions $f_{1}, f_{2}, \ldots, f_{n}$ are linearly independent, hence there are elements $x_{k}$ for $k=1,2, \ldots, n$ in $K$ such that the matrix $\left(f_{j}\left(x_{k}\right)\right)_{j, k=1}^{n}$ is regular. We have

$$
f_{i}\left(x_{k} * y\right)=\sum_{j=1}^{n} c_{i, j}(y) f_{j}\left(x_{k}\right)
$$

for each $y$ in $K$ and $k=1,2, \ldots, n$. For any fixed $i$ this is an inhomogeneous system of linear equations for the unknowns $c_{i, j}(y)(j=1,2, \ldots, n)$ with regular fundamental matrix, hence, by Cramer's rule, $c_{i, j}$ is a linear combination of translates of $f_{i}$, hence $c_{i, j}$ belongs to $V(i, j=1,2, \ldots, n)$.

Going back to equation (2) and using the associativity of convolution we infer that

$$
\sum_{j=1}^{n} c_{i, j}(z) f_{j}(x * y)=\sum_{j=1}^{n} c_{i, j}(y * z) f_{j}(x),
$$


or

$$
\sum_{j=1}^{n} c_{i, j}(z) \sum_{l=1}^{n} c_{j, l}(y) f_{l}(x)=\sum_{j=1}^{n} c_{i, j}(y * z) f_{j}(x)
$$

holds for each $x, y, z$ in $K$. This is equivalent to

$$
\sum_{k=1}^{n} \sum_{j=1}^{n} c_{i, j}(z) c_{j, k}(y) f_{k}(x)=\sum_{k=1}^{n} c_{i, k}(y * z) f_{k}(x) .
$$

By the linear independence of the $f_{k}$ 's we have

$$
\sum_{j=1}^{n} c_{i, j}(z) c_{j, k}(y)=c_{i, k}(y * z)=c_{i, k}(z * y)
$$

for each $y, z$ in $K$. Let $C(x)$ be the matrix $\left(c_{i, j}(x)\right)_{i, j=1}^{n}$, then from $(6)$ it follows

$$
C(x * y)=C(x) \cdot C(y)
$$

for each $x, y$ in $K$. In particular, the matrices $C(x)$ are commuting for different $x$ 's. It follows that there exists a nonsingular matrix $S$ such that the matrix $T(x)$ defined by

$$
T(x)=S^{-1} \cdot C(x) \cdot S
$$

is lower triangular for each $x$ in $K$. On the other hand, the entries of $T(x)$ are linear combinations of the $c_{i, j}$ 's, hence they belong to $V$. Further we have for each $x, y$ in $K$ :

$$
\begin{gathered}
T(x * y)=S^{-1} \cdot C(x * y) \cdot S=S^{-1} \cdot C(x) \cdot C(y) \cdot S= \\
\quad=S^{-1} \cdot C(x) \cdot S \cdot S^{-1} \cdot C(y) \cdot S=T(x) \cdot T(y) .
\end{gathered}
$$

Suppose that $T(x)=\left(t_{i, j}(x)\right)_{i, j=1}^{n}$, then using the fact that $T(x)$ is lower triangular we have that

$$
t_{i, j}(x * y)=\sum_{k=j}^{i} t_{i, k}(x) \cdot t_{k, j}(y)
$$

holds for $j=1,2, \ldots, i$ and for each $x, y$ in $K$. If we put $j=i$ we get

$$
t_{i, i}(x * y)=t_{i, i}(x) \cdot t_{i, i}(y)
$$

for $i=1,2, \ldots, n$ and for each $x, y$ in $K$, which means that the functions $t_{i, i}$ $(i=1,2, \ldots, n)$ are exponentials in $V$ and the theorem is proved.

\section{References}

[1] L. Székelyhidi, Spectral Analysis and Spectral Synthesis on Polynomial Hypergroups, Monatshefte Math., 141(1), (2004), 33-43.

[2] L. Székelyhidi, Spectral Synthesis on Multivariate Polynomial Hypergroups, Monatshefte Math., 153, (2008), 145-152. 